\title{
Utilización de la ultrasonografía para determinar el estado genital en la sincronización e inseminación artificial en vacas
}

\author{
Konrad, J.L. ${ }^{1,2}$; Crudeli, G.A. ${ }^{1}$ \\ ${ }^{1}$ Cátedra de Teriogenología, Facultad de Ciencias Veterinarias, Universidad Nacional del Nordeste, Sargento \\ Cabral 2139, Corrientes (3400), Argentina, Tel/Fax 0379-4425753; ${ }^{2}$ Consejo Nacional de Investigaciones \\ Científicas y Técnicas. (CONICET, Argentina).E-mail: konradjl@hotmail.com
}

\begin{abstract}
Resumen
Konrad, J.L.; Crudeli, G.A.: Utilización de la ultrasonografía para determinar el estado genital en la sincronización e inseminación artificial en vacas. Rev. vet. 29: 1, 18-21, 2018. El objetivo del trabajo fue evaluar el uso de tratamientos de control del ciclo estral, determinando el estado genital mediante ultrasonografía, sobre la eficiencia de detección y distribución de celos, y la preñez en vacas inseminadas artificialmente. El trabajo se realizó con 380 vacas. Los vientres fueron clasificados por medio de ultrasonografía según las estructuras ováricas y asignadas a cada tratamiento. En el tratamiento 1 se incluyeron vacas que presentaron cuerpo lúteo; se aplicó una dosis de prostaglandina y se realizó detección de celos e inseminación artificial por siete días. Para el tratamiento 2 se utilizaron las vacas que poseían folículos $\geq 8 \mathrm{~mm}$ de diámetro, se aplicó una dosis de hormona liberadora de gonadotrofinas, siete días después se colocó una dosis de prostaglandina y se procedió a la detección de celos e inseminación artificial por siete días. En el tratamiento 3 se realizó inseminación artificial a tiempo fijo con vacas que tenían folículos $\geq 8 \mathrm{~mm}$ o cuerpo lúteo. El tratamiento 4 se llevó a cabo con vacas que presentaron folículos $\geq 8 \mathrm{~mm}$ o cuerpo lúteo, se realizó inseminación artificial a celo detectado durante 21 días sin aplicación alguna de drogas. A los 30 días de finalizadas las inseminaciones se realizó diagnóstico de gestación por ultrasonografía para determinar estados de preñez. La evaluación de la detección de los celos fue del 81,8\%, 84,5\% y $46 \%(\mathrm{p}<0,05)$ para los tratamientos 1,2 y 4 respectivamente. Los porcentajes de concepción fueron del $59,26 \% ; 65,59 \% ; 34,02 \%$ y $65,22 \%(\mathrm{p}<0,05)$ y la preñez por inseminación fue de $48,48 \%, 55,45 \%, 34,02 \%$ y $30 \%$ (p $>0,05$ ), para los tratamiento $1,2,3$ y 4 respectivamente. El uso de tratamientos hormonales permitió mejorar el porcentaje de vacas detectadas en celo y la preñez por inseminación fue mayor en los tratamientos donde se inseminó a celo detectado.
\end{abstract}

Palabras clave: bovinos, ciclicidad, celo, inseminación, ultrasonografía.

\begin{abstract}
Konrad, J.L.; Crudeli, G.A.: Using ultrasound to determine the genital state in the synchronization and artificial insemination in beef cows. Rev. vet. 29: 1, 18-21, 2018. The objective of the study was to evaluate the use of estrous control treatments, determining genital status by ultrasonography, efficiency of detection and distribution of heat, and pregnancy in artificially inseminated cows. The work was carried out with 380 females. The cows was classified by ultrasonography according to the present ovarian structures and assigned to each treatment. In the treatment 1 were included cows that presented corpus luteum; was applied a dose of prostaglandin and from the next day was held the heat detection and artificial insemination for seven days. For the treatment 2 were used cows who had follicles $\geq 8$ millimeters of diameter, was applied a dose of gonadotrifins releasing hormone, seven days after was applied a dose of prostaglandin and proceeded to the heat detection and artificial insemination for seven days. In the treatment 3 was performed the fixed-time artificial insemination with cows who had follicles $\geq 8$ millimeters or corpus luteum. The treatment 4 or control was made with cows who had follicles $\geq 8$ millimeters or corpus luteum, was performed artificial insemination with heat detection during twenty one days without the application of some drugs. The heat detection rate was $81,8 \%, 84,5 \%$ and $46 \%$ for treatments 1,2 and 4 respectively $(\mathrm{p}<0,05)$. The conception rates were $59,26 \% ; 65,59 \% ; 34,02 \%$ and $65,22 \%(\mathrm{p}<0,05)$ for treatments $1,2,3$ and 4 , the pregnancy for insemination in each treatment were $48,48 \%$, $55,45 \%, 34,02 \%$ and $30 \%(p>0,05)$ respectively. The use of hormonal treatments improved
\end{abstract}


the percentage of cows detected in estrus, and pregnancy by insemination was higher in treatments where detected estrus and inseminated.

Key words: cows, cyclicity, heat, insemination, ultrasonography.

\section{INTRODUCCIÓN}

Los programas de manejo ganadero están orientados a obtener óptimos parámetros reproductivos, entre ellos una reducción del intervalo entre partos, buscando obtener una máxima eficiencia para garantizar el retorno económico ${ }^{3}$. Una de las principales limitantes para lograr este objetivo es la dispersión de los partos a través del año, lo que produce lotes de terneros menos uniformes ${ }^{8}$.

La detección de hembras que se encuentran en celo es uno de los aspectos más importantes en los programas de inseminación artificial (IA) ya que a partir de dicha manifestación se planifica el momento de la siembra del semen. El método más usado es el de observación visual, que si bien es una técnica simple y práctica, puede generar errores debido a su mala implementación ${ }^{9}$.

El avance en el conocimiento de la fisiología reproductiva de los bovinos, especialmente en lo referente a las características del desarrollo folicular, ha contribuido al progreso de protocolos de inseminación artificial a tiempo fijo (IATF) ${ }^{12}$. Realizando un examen ginecológico pre-servicio mediante ultrasonografía, se logra determinar la situación reproductiva de cada hembra y esto permite aplicar el tratamiento más adecuado.

El descubrimiento de las prostaglandinas como mediadores de las acciones hormonales y especialmente la identificación de la prostaglandina F2 $\alpha$ (PGF2 $\alpha$ ) como agente luteolítico, marcaron un hito en el desarrollo de la biotecnología reproductiva en el control del ciclo estral ${ }^{6}$. La sincronización con PGF2 $\alpha$, es exitosa cuando los animales son inseminados con detección de celo ${ }^{2}$. Sin embargo, éste no está sincronizado con precisión cuando se ha utilizado una sola dosis de PGF $2 \alpha^{18}$.

La administración exógena de hormona liberadora de gonadotrofinas $(\mathrm{GnRH})$ actúa indirectamente a través de la pituitaria anterior para iniciar la ovulación. Los efectos de la GnRH se observan a las 2-4 horas de la inyección, a través de un aumento de las concentraciones periféricas de hormonas folículo-estimulante y luteinizante ${ }^{23}$. En consecuencia, la utilización de agonistas de GnRH en combinación con un agente luteolítico ha permitido sincronizar el crecimiento folicular y la ovulación sin comprometer la fertilidad ${ }^{1,26}$.

La IATF obtiene resultados menores a los de la inseminación con celo detectado (IACD) ${ }^{15}$, razón por la cual se utilizan protocolos de control del ciclo estral que permiten realizar la IA sobre celo detectado.

El objetivo del trabajo fue evaluar el uso de tratamientos de control del ciclo estral, determinando el estado genital mediante ultrasonografía, sobre la efi- ciencia de detección y distribución de celos, así como la preñez en vacas inseminadas artificialmente.

\section{MATERIAL Y MÉTODOS}

La investigación se realizó en un establecimiento dedicado a la cría bovina, ubicado al sur de la localidad de Empedrado, Provincia de Corrientes, Argentina. Se utilizaron 380 vientres bovinos de la raza Brangus, variedades negro y colorado, categoría vacas secas, con un promedio de $5,39 \pm 1,88$ años de edad, una condición corporal de 4,16 $\pm 5,87$ (escala: 1-emaciada, 9-excesivamente gorda ${ }^{14}$ ), con un perímetro torácico de $174,29 \pm 55,71 \mathrm{~cm}$ y un peso corporal de $370,98 \pm 8,60 \mathrm{~kg}$.

Se tomaron muestras de semen de cada partida utilizada para la IA y se analizaron en microscopio para evaluar su motilidad y vigor, mediante el test de termo resistencia. El semen utilizado tuvo como mínimo un $25 \%$ de células móviles con un vigor de 3 (escala de 1 a 5) inmediatamente después del descongelado y un $15 \%$ de células móviles con vigor de 2 luego de 2 horas de incubación a $37^{\circ} \mathrm{C}^{4}$.

Los vientres fueron clasificados por medio de ultrasonografía según las estructuras ováricas predominantes y asignados a cada tratamiento al inicio del servicio. Animales que tenían como estructura ovárica predominante folículos con un diámetro $\leq 7 \mathrm{~mm}$ y no presentaban cuerpo lúteo (CL) se destinaron a servicio con toros y no se utilizaron para el trabajo $(n=57)$.

En el tratamiento 1 fueron incluidas vacas que al momento de la ecografía presentaron CL; se aplicó una dosis de PGF $2 \alpha$ (enantiómero dextrógiro del cloprostenol, $2 \mathrm{ml}$ por dosis, concentración de $0,075 \mathrm{mg} / \mathrm{ml}$ ), por vía intramuscular y a partir del día siguiente se realizó IACD por el sistema AM-PM por siete días ( $\mathrm{n}=66$ ).

Para el tratamiento 2 se utilizaron las vacas que a la ultrasonografía tenían en sus ovarios folículos $\geq 8 \mathrm{~mm}$ de diámetro, se aplicó una dosis de un análogo sintético de la hormona GnRH (acetato de buserelina, 2,5 $\mathrm{ml}$ por dosis, concentración de $0,0042 \mathrm{mg} / \mathrm{ml}$ ), por vía intramuscular). Siete días después se administró una dosis de PGF $2 \alpha$ y se procedió como en el tratamiento anterior a la IACD por siete días ( $\mathrm{n}=110)$.

En el tratamiento 3 se realizó IATF con vacas que tuvieron al momento de la ecografía folículos $\geq 8$ milímetros o CL. El protocolo utilizado fue el denominado Ovsynch $^{21}(n=97)$.

El tratamiento 4 (control) se llevó a cabo con vacas que al momento de la ultrasonografía presentaron folículos $\geq 8$ milímetros o $\mathrm{CL}$, se realizó IACD durante 21 días sin aplicación alguna de drogas $(n=50)$. En los tratamientos 1,2 y 4 se evaluó el porcentaje de celo 
detectado y la distribución de los mismos. A los 30 días de finalizadas las inseminaciones se realizó diagnóstico de gestación por ultrasonografía.

Para evaluar relación entre las variables presentación de celos, concepción (preñadas/inseminadas) y porcentajes de preñez por inseminación (preñadas/total del rodeo), se utilizó el estadístico de Chi cuadrado con un nivel de significancia igual al $5 \%{ }^{10}$.

\section{RESULTADOS Y DISCUSIÓN}

\section{Detección de celos}

La tasa de detección de celos fue del 81,8\% (54/66), $84,5 \%(93 / 110)$ y $46 \%(23 / 50)$, para los tratamientos 1 , 2 y 4 respectivamente. Fueron encontradas diferencias significativas entre los tratamientos 1 y 2 con respecto al tratamiento $4(\mathrm{p}<0,05)$, pero no entre los dos primeros.

En cuanto a la distribución de los celos, tanto en los tratamiento 1 como 2 se observaron vacas en celo desde el día 1 con aumento exponencial hasta el día 3, produciéndose un pico máximo a las 72 horas, para luego disminuir hasta el día 7 posterior a la aplicación de PGF2 $\alpha$. La distribución de los celos en los tratamientos 1,2 y 4 se ilustra en la Figura 1.

En vacas Bos taurus se reportaron detecciones de celos del 70 al 95\%, luego de una única dosis de PGF2 $\alpha$ ${ }^{24,27}$, resultados similares a los obtenidos en este trabajo. Sin embargo, respuestas de 46 a $62 \%$ de celo se observaron tras una sola dosis de PGF $2 \alpha$ en vacas $B o s$ indicus ${ }^{17,19}$. En otra investigación se observó una frecuencia de aparición de celos del 43; 83,6 y $100 \%$ en vacas, cuando se administró PGF2 $\alpha$ entre los días 5-7, 8-11 y 12-15 del ciclo, respectivamente ${ }^{27}$, dentro de estos grupos el pico del celo se observó a las 59, 70 y 72 horas, debiéndose señalar que la longitud del intervalo desde la inyección de PGF2 $\alpha$ hasta la ovulación depende del estadio del folículo dominante al momento del inicio del tratamiento ${ }^{16}$.

En vacas y vaquillas Nelore se informó una respuesta de celo de solo 46,4 y 33,3\% después de dos dosis de PGF $2 \alpha$ con 11 días de intervalo ${ }^{20}$, en tanto que en vaquillas cruza cebú sincronizadas con doble dosis

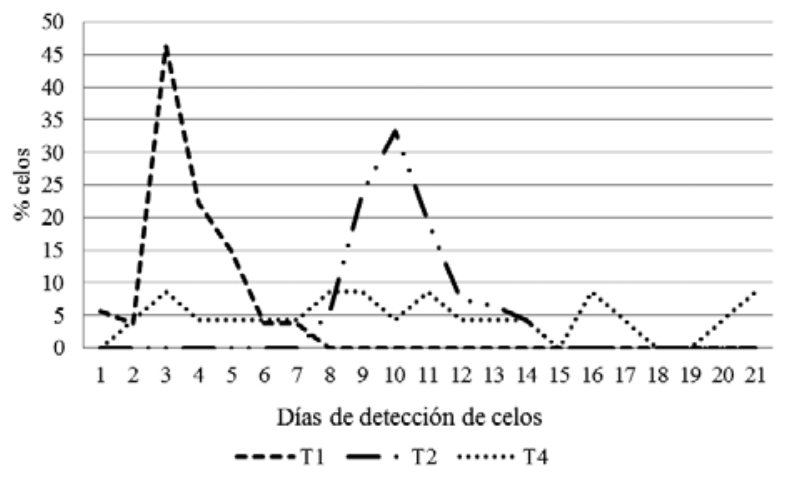

Figura 1: Distribución de celos en días del protocolo, para los tratamientos 1,2 y 4 . de PGF2 $\alpha$ aplicadas cada 12 días detectaron el 80,4\% de celos ${ }^{22}$. En trabajos en que aplicaron GnRH y 7 días más tarde PGF2 $\alpha$, la tasa de detección de celo varió del 70 al $83 \%^{7,25,26}$, resultados algo inferiores a los hallados en este trabajo.

\section{Preñez}

Los porcentajes de concepción fueron del 59,26\% (32/54), 65,59\% (61/93), 34,02\% (33/97) у 65,22\% $(15 / 23)$ para los tratamientos $1,2,3$ y 4 respectivamente. Las diferencias de proporciones entre 1, 2 y 4 no fueron significativas $(p>0,05)$, pero fueron significativas entre estos tres tratamientos con respecto al tratamiento $3(p<0,05)$. Los porcentajes de preñez por inseminación (preñadas/total del rodeo) en cada tratamiento fueron $48,48 \%, 55,45 \%, 34,02 \%$ y $30 \%$ respectivamente $(p>0,05)$, siendo superior en los dos primeros.

$\mathrm{Al}$ evaluar la concepción según la estructura ovárica al inicio del tratamiento, se encontró que las vacas que tenían CL se preñaron más cuando la inseminación se realizó a celo detectado, en comparación con la IATF $(p<0,05)$. No se encontraron diferencias dentro de los tratamientos si las vacas presentaron folículos de 8 a $10 \mathrm{~mm}$ o folículos de $11 \mathrm{~mm}$ o mayores.

En trabajos donde se aplicaron GnRH y PGF $2 \alpha 7$ días más tarde, la tasa de preñez fue del 65 a $85 \%^{7,26}$ detectando celos por 4 días. Otros autores, trabajando con vacas de carne mestizas (Brahman x Hereford), detectaron celo luego de la aplicación de PGF2 $\alpha$ siete días después de una dosis de GnRH, obteniendo una preñez del $72,7 \%$ y con el protocolo Ovsynch la concepción fue de 42,8 y 9,1\%, inseminándose a las 16 o 24 horas después de las segunda dosis de GnRH ${ }^{13}$. Estos resultados son superiores a los obtenidos en nuestro trabajo, tanto en la concepción con detección de celo, como con el protocolo Ovsynch con IA a las 24 horas.

En tanto, información sobre el uso del protocolo Ovsynch en vacas de carne, muestran porcentajes de concepción variables, desde $33 \%{ }^{11}$ al $69 \%{ }^{23}$. En vaquillas cruza cebú del noreste argentino, utilizando el mismo protocolo, la preñez por inseminación fue del $27,6 \%{ }^{22}$. En otro trabajo, donde se evaluó la respuesta con el protocolo Ovsynch en vacas cebú secas y con

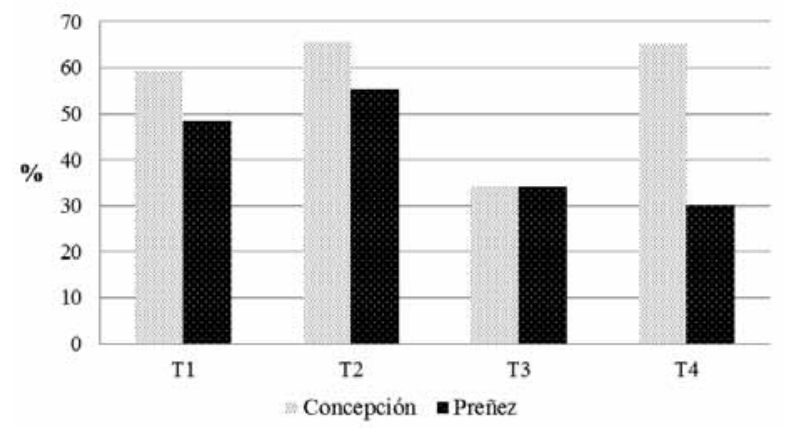

Figura 2: porcentajes de concepción (preñadas/inseminadas), y preñez (preñadas/total del rodeo) en cada tratamiento. 
ternero al pie, se obtuvieron tasas de preñez después de la IATF, que fueron del 42 al $48 \%{ }^{5}$.

De los resultados obtenidos en el presente trabajo se puede concluir que el uso de la ultrasonografía para determinar el estado genital permitió mejorar el porcentaje de vacas detectadas en celo. La preñez por inseminación artificial fue mayor en los tratamientos donde se realizó la sincronización de los celos y se inseminó a celo detectado.

\section{REFERENCIAS}

1. Adams GP, Matteri RL, Kastelic JP, Ko JC, Ginther OJ. 1992. Association between surges of follicle stimulating hormone and the emergence of follicular waves in heifers. J Reprod Fertil 94: 177-188.

2. Archbald LF, Tran T, Massey R, Klapstein E. 1992. Conception rates in dairy cows after timed-insemination and simultaneous treatment with gonadotropin releasing hormone and/or prostaglandin F2 $\alpha$. Theriogenology 37: 723-728.

3. Arthur GH, Noakes DE, Pearson H, Parkinson TJ. 1996. Veterinary reproduction and obstetrics, 7th. ed., Saunders Co., London, p. 226.

4. Barth AD. 1995. Evaluation of frozen semen by the veterinary practitioner. Proceeding Soc. Theriogenology, Meeting Kansas City, USA, p. 105-110.

5. Bo GA, Baruselli PS, Martínez MF. 2003. Pattern and manipulation of follicular development in Bos indicus cattle. Anim Reprod Sci 78: 307-326.

6. Bo GA, Cutaia L, Moreno D, Tríbulo HE. 2008. Sincronización de celos e inseminación artificial, $1^{\mathrm{a}}$ ed., Publ. Inst. Reprod. Anim. Córdoba, Argentina, p. 39-107.

7. Coleman DA, Bartol FF, Spencer TE, Floyd JG, Wolfe DF, Brendemuehl JP. 1991. Effects of a potent GnRH agonist on hormonal profiles, synchronization of estrus and fertility in beef cattle. J Anim Sci 69: 396.

8. Cutaia LE. 2011. Programas de IATF: análisis de costos e implementación. Rev Aman Rural 1: 58-63.

9. De la Sota RL. 2000. Detección de celos: cómo calcular su intensidad y exactitud. Rev Taurus 2: 19-27.

10. Di Rienzo JA, Casanoves F, Balzarini MG, Gonzalez L, Tablada M, Robledo CW. InfoStat versión 2015. Grupo InfoStat, FCA, Universidad Nacional de Córdoba, Argentina.

11. Geary TW, Whittier JC, LeFever DG. 1998. Effect of calf removal on pregnancy rates of cows synchronized with the Ovsynch or Co-Synch protocol. J Anim Sci 76: 278.

12. Ginther OJ, Kastelic JP, Knopf L. 1989. Composition and characteristics of follicular waves during the bovine estrous cycle. Anim Reprod Sci 20: 187-200.

13. Gutiérrez JC, Palomares R, Sandoval J, Ondíz A, Portillo G, Soto E. 2005. Uso de protocolo Ovsynch en el control del anestro posparto en vacas mestizas de doble propósito. Rev Cient FCV-LUZ 25: 7-13.

14. Herd DB, Sprott LR. 1986. Body condition, nutrition and reproduction of beef cows. Texas A\&M Univ. Ext. Bull. B-1526.
15. Kasimanickam R, Cornwell JM, Nebel RL. 2005. Fertility following fixed-time AI or insemination at observed estrus in Ovsynch and Heatsynch programs in lactating dairy cows. Theriogenology 63: 2550-2559.

16. Kastelic JP, Ginther OJ. 1990. Effect of day of prostaglandin F2 $\alpha$ treatment on selection and development of the ovulatory follicle in heifers. Anim Reprod Sci 23: 169-180.

17. Landivar C, Galina CS, Duchateau A, Navarro R. 1985. Fertility trial in Zebu cattle after a natural or controlled estrus with prostaglandin F2 alpha, comparing natural mating with artificial insemination. Theriogenology 23: 421-429.

18. Lauderdale JW, Seguin BE, Stellflug JN, Chenault JR, Thatcher WW, Vincent CK, Loyancano AF. 1974. Fertility of cattle following PGF2 $\alpha$ injection. J Anim Sci 38: 964-967.

19. Orihuela A, Galina CS, Escobar FJ, Riquelme E. 1983. Estrous behavior following prostaglandin F2 $\alpha$ injection in Zebu cattle under continuous observation. Theriogenology 19: 795-809.

20. Pinheiro OL, Barros CM, Figueiredo RA, Valle ER, Encarnacao RO, Padovani CR. 1998. Estrous behavior and the estrus-to-ovulation interval in Nelore cattle (Bos indicus) with natural estrus or estrus induced with prostaglandin F $2 \alpha$ or norgestomet and estradiol valerate. Theriogenology 49: 667-681.

21. Pursley JR, Mee MO, Wiltbank MC. 1995. Synchronization of ovulation in dairy cows using PGF $2 \alpha$ and GnRH. Theriogenology 44: 915-924.

22. Stahringer RC, Mastandrea O. 2005. Respuesta de vaquillas cruza cebú con distinto grado de desarrollo genital a la sincronización de celo con progestágenos, GnRH y/o prostaglandina. Foll. EEA INTA Colonia Benitez, p. 3-7.

23. Stevenson JS, Thompson KE, Forbes WL, Lamb GC, Grieger DM, Corah LR. 2000. Synchronizing estrus and (or) ovulation in beef cows after combinations of GnRH, norgestomet, and prostaglandin F2 $\alpha$ with or without timed insemination. J Anim Sci 78: 1747-1758.

24. Tanabe TY, Hann RC. 1984. Synchronized estrus and subsequent conception in dairy heifers treated with prostaglandin F2 alpha. Influence of stage of cycle at treatment. J Anim Sci 58: 805-811.

25. Thatcher WW, MacMillan KL, Hansen PJ, Drost M. 1989. Concepts for regulation of corpus luteum function by the conceptus and ovarian follicles to improve fertility. Theriogenology 31: 149-164.

26. Twagiramungu H, Guilbault LA, Proulx J, Dufour JJ. 1992. Synchronization of estrus and fertility in beef cattle with two injections of buserelin and prostaglandin. Theriogenology 38: 1131-1144.

27. Watts TL, Fuquay JW. 1985. Response and fertility of dairy heifers following injection with prostaglandin F $2 \alpha$ during early, middle, and late diestrus. Theriogenology 23: 655-661. 